\title{
Design of a plasmonic metasurface laser accelerator with a tapered phase velocity for subrelativistic particles
}

\author{
Doron Bar-Lev, ${ }^{1}$ R. Joel England, ${ }^{2}$ Kent P. Wootton, ${ }^{2}$ Weihao Liu, ${ }^{2}$ Avraham Gover, ${ }^{1}$ \\ Robert Byer, ${ }^{3}$ Ken J. Leedle, ${ }^{4}$ D. Black, ${ }^{4}$ and Jacob Scheuer ${ }^{1, *}$ \\ ${ }^{1}$ School of Electrical Engineering Tel Aviv University, Ramat Aviv, Tel-Aviv 69978, Israel \\ ${ }^{2}$ SLAC National Accelerator Laboratory, 2575 Sand Hill Road, Menlo Park, California 94025, USA \\ ${ }^{3}$ Department of Applied Physics, Stanford University, Stanford, California 94305, USA \\ ${ }^{4}$ Department of Electrical Engineering, Stanford University, Stanford, California 94305, USA
}

(Received 25 September 2018; published 11 February 2019)

\begin{abstract}
A metallic metasurface-based laser-driven particle accelerator for subrelativistic particles is proposed and studied theoretically. The metasurface consists of a nonperiodic array of nanoslits which focuses the field of the driving laser, utilizing the phenomenon of extraordinary plasmonic transmission, to maximize the acceleration gradient. In order to account for the actual change in the particles' velocity during their propagation through the structure, the separation between successive slits is not constant but rather optimized according to the expected trajectory of the particles. The metasurface laser accelerator (MLA) is designed for an ultrafast driving laser source operating at $2 \mu \mathrm{m}$ wavelength. An approximate analytical model verified by particle tracking simulations predicts a net average acceleration with a normalized acceleration gradient of 1.34 times the incident laser field. Compared to other laser-driven accelerator designs, the MLA provides substantially higher efficiency, due to the field enhancement associated with nanoantennas, and relaxed fabrication challenges (especially for subrelativistic particles). It is found that the output particle beam is microbunched, suggesting the possibility of using a short MLA structure as a prebuncher to improve the initial capture efficiency in a subsequent longer MLA device. The impact of space-charge effects is also studied, and the loaded gradient and optimal bunch charge are estimated.
\end{abstract}

DOI: 10.1103/PhysRevAccelBeams.22.021303

\section{INTRODUCTION}

Laser-driven particle accelerators are emerging as a unique and powerful approach for obtaining very large acceleration gradients, compared to conventional radio frequency (rf) accelerators, by utilizing commercially available ultrafast lasers and well-established nanofabrication methods. Acceleration gradients of several hundreds of $\mathrm{MeV} / \mathrm{m}$ have been demonstrated by dielectric laser accelerators (DLAs) employing $\mathrm{SiO}_{2}$ [1-5] and silicon [4,6-9] gratings for both relativistic and nonrelativistic electrons. More recently, it was shown theoretically that employing plasmonic nanostructures to realize metasurface laser accelerators (MLAs) could enable similarly high acceleration gradients with improved efficiency [10].

The underlying concept of laser-driven structure-based accelerators is to utilize the strong electric field of an ultrafast laser pulse to excite a suitable electromagnetic

*kobys@eng.tau.ac.il

Published by the American Physical Society under the terms of the Creative Commons Attribution 4.0 International license. Further distribution of this work must maintain attribution to the author(s) and the published article's title, journal citation, and DOI. mode in a photonic structure in order to accelerate charged particles. In order to synchronously accelerate a particle, the phase velocity of the electromagnetic accelerating mode must be matched to the instantaneous speed of the particle. As discussed in Sec. II, for slab-symmetric side-driven structures such as the one discussed here, the phase velocity is set by the use of periodic features along the acceleration axis fabricated into the structure. Achieving synchronism for highly relativistic particles is relatively simple, as the velocity of the particles does not change appreciably during the acceleration process. As a result, the corresponding periodicity (or unit cell length) is constant $[4,11,12]$. For subrelativistic electrons, on the other hand, the particles do change their velocity as they gain energy, thus necessitating tapered structures where the local periodicity varies gradually along the structure. The design of such tapered structures is not obvious, as it requires solving for the particles' velocity profile along the accelerator simultaneously with the electromagnetic problem. In addition, accelerating slower particles necessitates denser and smaller feature sizes, rendering the fabrication of such accelerators more challenging.

Although phase velocity tapering is a well-established approach in contemporary rf-based accelerators $[13,14]$, it has been scarcely studied in the context of laser-driven 
accelerators. Breuer and Hommelhoff used a fused silica grating structure for nonrelativistic electron acceleration and discussed the possibility of cascading such (discrete) structures with varying periodicity to improve synchronism [6]. It should be noted, however, that this structure operated at the third spatial harmonic of the fundamental mode, which relaxes the fabrication challenges and allows for subrelativistic phase velocity. Nevertheless, operating at a higher harmonic reduces the accelerating gradient. More recently, Niedermayer, Boine-Frankenheim, and Egenolf proposed a silicon-based chirped accelerator structure where the period is modified continuously according to the expected trajectory of the accelerated particles [15].

In this paper, we present and study a new MLA concept which is based on extraordinary transmission [16] through a metasurface comprising a nonperiodic array of slot nanoantennas. In contrast to most DLA and MLA structures presented previously $[1-10,12,15]$, the new design does not attempt to control the phase profile of the field in order to obtain net acceleration. Rather, the device produces a field enhancement with a flat phase profile at the positions in which the instantaneous vector of the electric field is aligned with the electron velocity. Consequently, it is straightforward to optimize the design to any desired initial particle energy and impinging field amplitude. Moreover, as shown below, the design yields a net acceleration of the particles (in contrast to energy modulation, which is commonly presented by laser-based accelerators) as well as microbunching of the particles due to the nonperiodic nature of the structure.

The tapering scheme presented here can be implemented also in DLAs. Nevertheless, the MLA approach offers several inherent advantages which render it highly attractive, especially for subrelativistic particle acceleration: (i) ease of fabrication-metallic metasurfaces are ultrathin (tens of nanometers) compared to their dielectric counterpart and, consequently, the realization of the small features needed for slower particles is less challenging; (ii) the field enhancement property associated with metallic nanostructures provides a higher "structure factor", or ratio of axial gradient to the driving electric field strength, which improves the efficiency of converting the power of an incident laser field into particle energy.

The paper is organized as follows. In Sec. II, we present an MLA structure utilizing a discrete phase velocity matching approach and extract the field distribution and acceleration gradient. In Sec. III, we present the background theory of resonant acceleration to provide a heuristic model of the longitudinal dynamics in the MLA. In Sec. IV, we use particle tracking simulations to study the acceleration of electron beams in the MLA structure in Sec. II and compare the results to those of the continuous model described in Sec. III. In Secs. V and VI, we study, respectively, microbunching and space-charge effects, and in Sec. VII, we summarize our results and present some concluding remarks. (a)

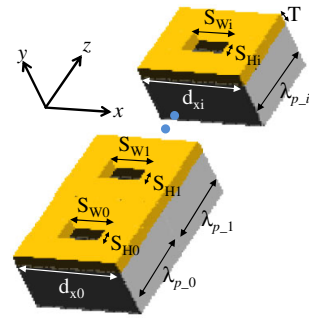

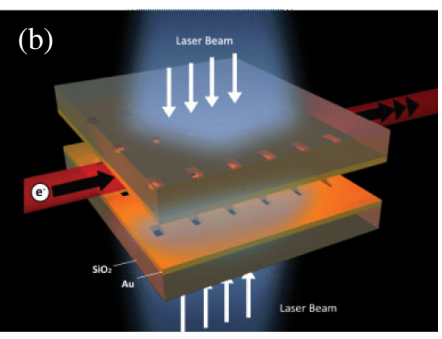

FIG. 1. (a) Schematic of a single row of slot antenna unit cells in the metasurface; (b) schematic of the MLA formed by two parallel metasurfaces, symmetrically driven by cophased laser pulses incident from the top and bottom.

\section{TAPERED STRUCTURE DESIGN}

The MLA concept is based on strong field enhancement obtained at the plasmonic resonance wavelength of a metasurface composed of slits in a 40-nm-thick metallic film on a fused silica substrate, as shown in Fig. 1(a). Figure 1(b) depicts the schematic of the assembled accelerator structure, which consists of two such metasurfaces separated by a gap of width $g$ ( $200 \mathrm{~nm}$ here) through which the electron beam propagates (the vacuum chamber).

A pair of coherent driving laser pulses illuminates the structure from the top and bottom symmetrically, propagating through the substrate at normal incidence to the particle acceleration axis. As discussed in more detail in Sec. III, this symmetric illumination reduces transverse deflection forces and produces a nearly uniform acceleration force across the channel. If the dimensions of the slots shown in Fig. 1(a) are set such that their resonance wavelength is equal to the wavelength of the driving laser, they can efficiently focus the impinging light through the slits and generate a region of enhanced electric field in the vacuum channel. This phenomenon is known as extraordinary transmission [16]. The strong field obtained in the vacuum channel is utilized for accelerating the particles.

Continuous tapering of such a structure to match its phase velocity to the increasing energy of a subrelativistic beam is extremely challenging, especially at optical frequencies. A more realistic solution is to divide the accelerator into several sections, as proposed in Refs. [7,17], with each successive section optimized for the targeted particle energy at that location. However, the change in particle velocity within each section will cause the particles to slip in phase. This slippage can be greatly reduced by tapering the accelerator in steps of a single cell, leading to chirped structures [15]. Consequently, $\gamma_{i+1}$, the average Lorenz factor of the beam in the $i+1$ cell, can be calculated recursively from the $i$ th cell factor $\left(\gamma_{i}\right)$ and the energy gain in each cell. By utilizing the definition of the acceleration gradient at each cell, $G_{i}$, this relation corresponds to

$$
\gamma_{i+1}=\gamma_{i}+\frac{G_{i} \lambda_{p, i}}{m c^{2}}
$$




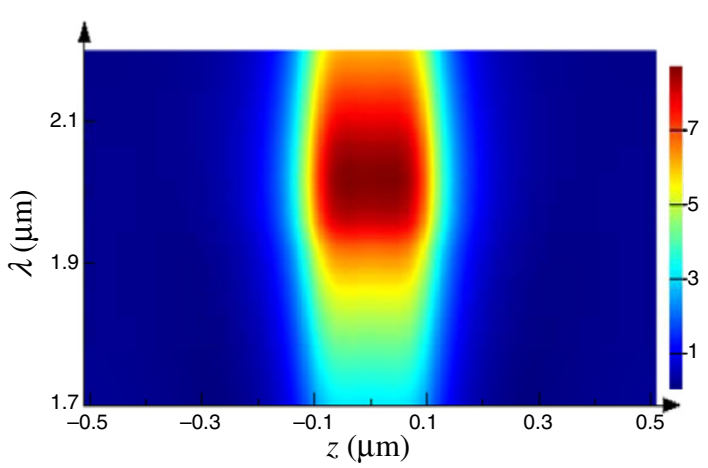

FIG. 2. Color contour plot of the electric field amplitude normalized to that of the driving field as a function of the wavelength and longitudinal position within a single unit cell. Periodic boundary conditions in $z$ and $x$ have been assumed for the calculation of the field.

where $\lambda_{p, i}$ is the length of the $i$ th cell which stems from the synchronicity condition in that cell, i.e., $\lambda_{p, i}=\beta_{i} \lambda$, where $\lambda$ is the laser wavelength and $\beta_{i}=\sqrt{1-1 / \gamma_{i}^{2}}$. From Eq. (1), a recursive relation between the lengths of consecutive cells can be calculated:

$$
\lambda_{p, i+1}=\lambda \sqrt{1-\left(\gamma_{i}+\frac{G_{i} \lambda_{p, i}}{m c^{2}}\right)^{-2}} .
$$

Figure 2 shows a color contour plot of the electric field amplitude (normalized to incident field $E_{0}$ ) along the axis of a single cell of the multicell structure shown in Fig. 1. The fields are calculated using a finite difference time domain (FDTD) analysis. The chosen slot dimensions $\left(S_{W}=600 \mathrm{~nm}\right.$ and $\left.S_{H}=200 \mathrm{~nm}\right)$ provide maximal field enhancement at a wavelength $\lambda=2 \mu \mathrm{m}$. It should be noted that, for this plot, periodic boundary conditions in $z$ and $x$ have been employed with periods of $d_{x}=1.1 \mu \mathrm{m}$ and $\lambda_{p}=1.35 \mu \mathrm{m}$, respectively. The impact of the difference between the adjacent cells of the tapered structure on the resonance and field profile in each cell is negligible. On the other hand, the impact of the tapering on the particle trajectory is important as discussed further in Sec. III.

Extending this optimization to the multicell design in Fig. 1 requires that the length of each cell be adjusted in order to maintain continuous acceleration. The design of a tapered MLA requires a determination of the optimal slit dimensions and relative positions along the acceleration direction ( $z$ axis in Fig. 1). The dimensions of the slits are optimized for maximal field enhancement, and the $z$ separations between them are chosen to match the phase velocity of the accelerating mode in the channel to the energy of the resonant particle. Because of the tapering of the "unit-cell" length, the actual field enhancement may deviate from the values in Fig. 2. However, due to the relatively small difference in the length of the unit cells, the deviations are minor.

Figure 3 shows the unit-cell lengths and $\lambda_{p, i}$ for a 17-cell MLA designed for an initial kinetic energy of $79 \mathrm{keV}$ and

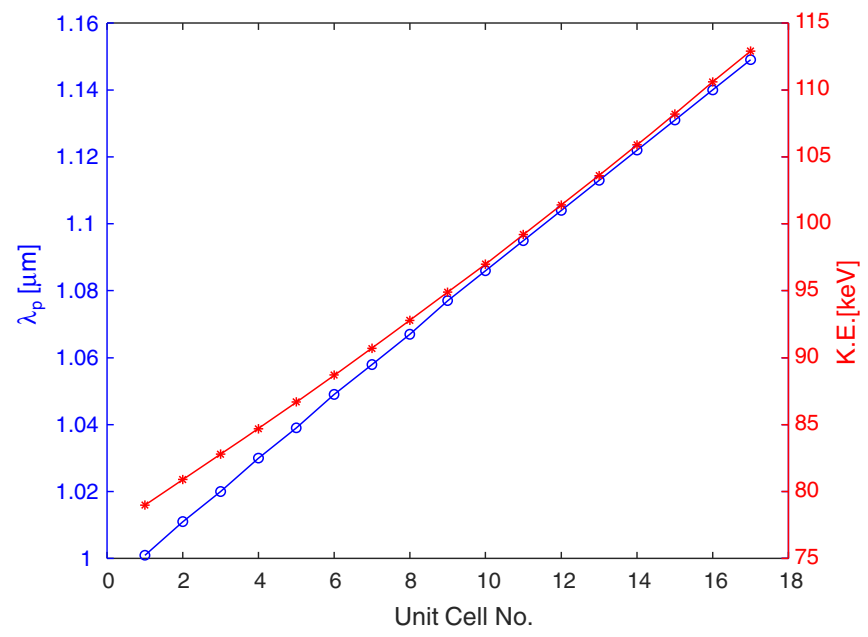

FIG. 3. Unit-cell length (blue, circles) and corresponding kinetic energy (KE) (red, stars) for a 17-cell tapered MLA designed for an initial energy of $79 \mathrm{keV}$ and an incident field amplitude of $0.5 \mathrm{GV} / \mathrm{m}$.

incident laser electric field amplitude of $E_{0}=0.5 \mathrm{GV} / \mathrm{m}$ from each side (corresponding to an average acceleration gradient of $G=1.4 \mathrm{GeV} / \mathrm{m}$ ). This incident field amplitude was chosen based upon preliminary laser damage studies conducted on a sequence of single-sided prototype MLA arrays manufactured according to the target parameters shown in Fig. 3 as described in Appendix C. A complete table of the unit-cell dimensions and the corresponding particle velocities and kinetic energies is found in Table I in Appendix A. We note that the lengths of the unit cells, $\lambda_{p, i}$, increase from $1 \mu \mathrm{m}$ (corresponding to normalized particle velocity $\beta=v / c=0.5$ ) to $1.15 \mu \mathrm{m}$ (corresponding to $\beta=0.575$ ). The dimensions of the slits are identical for all cells.

Figure 4 depicts the $z$ component of the electric field at the channel center of an enclosed (dual layer) MLA

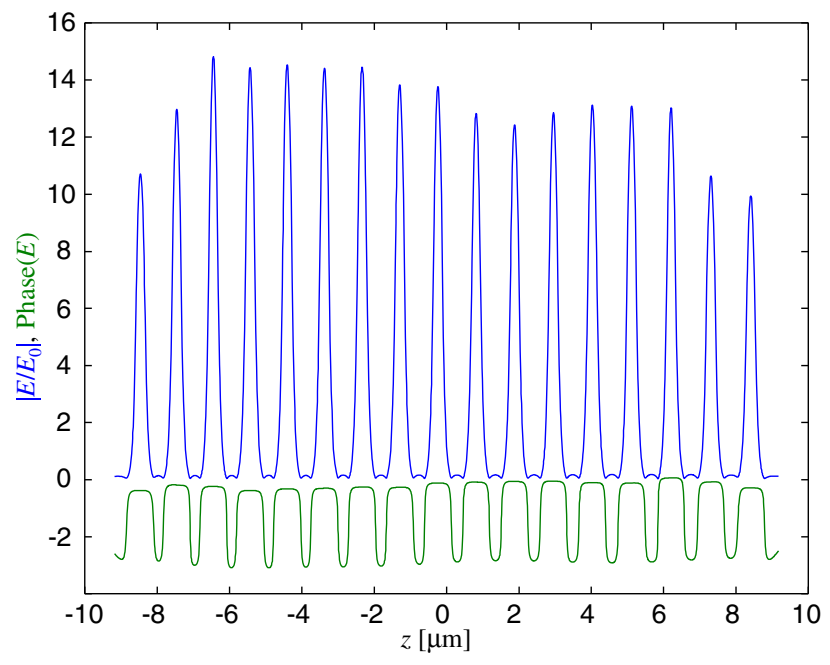

FIG. 4. $E_{z}$ component of the field at the center of the enclosed tapered MLA structure. Blue, magnitude; green, phase. 
structure comprising 17 cells, calculated by FDTD simulations for a driving laser wavelength of $2 \mu \mathrm{m}$. As expected, peak electric field intensity occurs at the positions of the slits. The slight variations in the peak field intensity between cells are attributed to edge effects due to the finite size of the device.

\section{RESONANT PARTICLE MODEL}

The longitudinal electromagnetic field in the accelerating channel of a periodic dual-driven planar symmetric structure can be expressed in Floquet form as a superposition of spatial harmonics (see also Appendix B). Adopting the coordinate system in Fig. 1, where $z$ is the propagation axis of the particles (as well as the periodicity direction) and $y$ is the propagation direction of the driving laser (assuming normal illumination), the nonvanishing electric and magnetic field components in the vacuum channel can be written as

$$
\begin{gathered}
E_{y}=-E_{0} \sum_{n=-\infty}^{\infty} \frac{k_{n}}{\Gamma_{n}} a_{n} \sinh \left(\Gamma_{n} y\right) e^{i k_{n} z} e^{-i \omega t}, \\
E_{z}=-i E_{0} \sum_{n=-\infty}^{\infty} a_{n} \cosh \left(\Gamma_{n} y\right) e^{i k_{n} z} e^{-i \omega t}, \\
B_{x}=E_{0} \sum_{n=-\infty}^{\infty} \frac{\omega}{c} \frac{1}{\Gamma_{n}} a_{n} \sinh \left(\Gamma_{n} y\right) e^{i k_{n} z} e^{-i \omega t},
\end{gathered}
$$

where $E_{0}$ is the incident field amplitude, $\omega$ is the angular frequency of the laser, $a_{n}$ is a geometry-dependent coupling coefficient, $\Gamma_{n}=\sqrt{k_{n}^{2}-(\omega / c)^{2}}$ is the exponential decay constant in the $y$ direction, and $k_{n}=k_{0}+n k_{p}$ is the longitudinal wave number of the $n$th space harmonic, $k_{p}=2 \pi / \lambda_{p}$, where $\lambda_{p}$ is the longitudinal periodicity and $k_{0}$ is the projection of the incident laser field wave number in the first Brillouin zone. A more detailed description is given in Appendix B. For a laser field at normal incidence to the $x-z$ plane, $k_{0}=0$. It should be emphasized that the structure discussed in Sec. II is not periodic but rather consists of cells of increasing length. This is in order to keep the accelerating particles in resonance with the driving laser field. However, the length of the cells changes adiabatically in $z$, and $\lambda_{p}(z)$ should be considered as the local periodicity of the structure.

A heuristic description of the longitudinal and transverse dynamics (in the absence of wakes and space charge) in the tapered MLA structure can be provided by the resonant particle model adapted from conventional particle accelerators $[18,19]$ and using the analytical formulation of the fields in Eqs. (3)-(5). The unloaded acceleration gradient $G_{0}=e E_{0} a_{r}$ of the resonant mode $(n=r)$ can be calculated directly from Eq. (3), where $e$ is the electron charge. The hyperbolic cosine dependence of $E_{z}$ can be seen to approach unity (i.e., a uniform field) in the limit where the vacuum gap $g \ll \Gamma_{r}^{-1}$. In this limit, the Lorentz force components (taking the real part of the fields) yield the following simplified forms:

$$
F_{z}=G_{0} \sin \phi, \quad F_{y}=G_{0} y k_{r}\left(\beta \beta_{r}-1\right) \cos \phi,
$$

where $\phi=k_{r} z-\omega t$ is the phase of the particle (with respect to the driving EM wave), satisfying $d \phi / d z=$ $k_{r}-\omega(d t / d z)$, and we have taken only the mode $n=r$ which corresponds to the desired phase velocity $\beta_{r}$ (normalized to speed of light $c$ ) for matching to the resonant particle. The relativistic equations of motion can thus be described by the following coupled differential equations:

$$
\begin{gathered}
\frac{d \phi}{d z}=\frac{\omega}{c}\left(\frac{1}{\beta_{r}}-\frac{1}{\beta}\right) ; \quad \frac{d \gamma}{d z}=\frac{\beta_{r}}{\beta} \frac{\omega}{c} \alpha_{0} \sin \phi, \\
\frac{d^{2} y}{d z^{2}}=\frac{k_{r} y}{\gamma \beta^{2}} \frac{\omega}{c} \alpha_{0}\left(\beta_{r}^{2}-1\right) \cos \phi-K^{2} y,
\end{gathered}
$$

where $\alpha_{0}=G_{0} c / \omega m c^{2}$ is a unitless normalized acceleration gradient and $K$ is an assumed external focusing term for particle confinement. Although the overall phase of the fields is arbitrary, we have chosen the convention that a phase of $\phi=\pi / 2$ corresponds to the peak accelerating force in $z$, for consistency with common usage in accelerator textbooks and seminal papers on rf photoinjector physics. When utilizing a laser to accelerate charged particles, the corresponding normalized energy gain per laser period $\lambda$ is of the order of $\alpha_{0} \approx 0.001$ for accelerating gradients of the order of $1 \mathrm{GeV} / \mathrm{m}$, consistent with recent demonstrations of laser-driven acceleration $[2,3,6,7]$. This value of $\alpha_{0}$ is 3 orders of magnitude smaller than that of a conventional radio frequency (rf) electron photoinjector for which typically $\alpha_{0} \approx 1$. This is due to the 4 orders of magnitude reduction in the operating wavelength. However, it is similar in magnitude to that of an rf proton accelerator. Therefore, as is conventionally done in proton linacs, maintaining phase synchronicity with the accelerating wave and capturing subrelativistic particles requires that the fundamental structural wave number $k_{r}(z)$ of the laser accelerator will vary according to the particle trajectory, resulting in a tapered structure $[13,14]$.

Taking the resonant mode to be the fundamental $(r=1)$, the velocity of the resonant particle is related to the $z-$ varying wave number via $k_{r}(z)=k_{0} / \beta_{r}(z)=2 \pi / \lambda_{p}(z)$. Assuming that the structure is designed for a constant unloaded acceleration gradient $G_{0}$ and that $\alpha_{0} \ll 1$ (as expected for laser accelerators), the energy of the resonant particle at position $z$ is given by $\gamma_{r}(z)=\gamma_{0}+\gamma^{\prime} z$, where $\gamma_{0}$ is the injection energy and $\gamma^{\prime}=k_{0} \alpha_{0} \sin \phi_{r}$. We can consider this resonant particle for which $\beta=\beta_{r}$ as the particle whose velocity profile defines the design gradient and wave number tapering of the structure. The synchronicity 

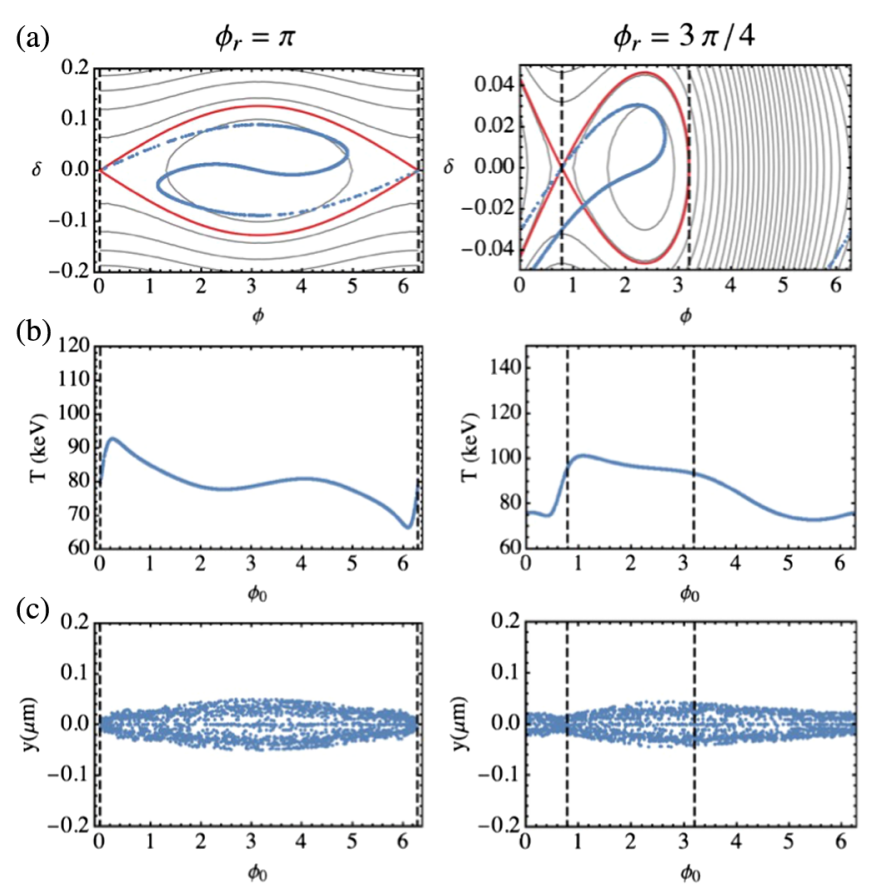

FIG. 5. Plots showing the (a) final longitudinal particle phase space superimposed with the Hamiltonian separatrix (in red), (b) energy spectrum as a function of the injection phase $\phi_{0}$ with a stable accelerating region marked by vertical lines, and (c) transverse position $y$ vs injection phase. Plots on left and right correspond to resonant phases $\phi_{r}=\pi$ and $\phi_{r}=3 \pi / 4$, respectively. The left column corresponds to a nontapered structure, while the right column corresponds to a tapered structure.

condition between the particle velocity and the driving laser may be expressed as follows [5]:

$$
\beta=\frac{\lambda_{p}}{n \lambda} \equiv \beta_{r} .
$$

Typically, the dominant mode is the fundamental one $(n=1)$. In this case, the synchronicity condition corresponds to $\lambda_{p}(z)=\lambda \beta(z)$, and thus, without the loss of generality, this relation applies for the remainder of the analysis.

As a concrete example, we consider the solutions of a continuous model corresponding to the MLA structure described in Sec. II. Figure 5 depicts the solutions to Eqs. (7) and (8) for the propagation of a collection of injected particles uniformly distributed over all phases and over a vertical range $\Delta y=100 \mathrm{~nm}$, for two different resonant phases $\phi_{r}=\pi$ and $3 \pi / 4$ on the left and right, respectively. The initial energy of the particles is $79 \mathrm{keV}$, and an external focusing force $K^{2}=1 \mu \mathrm{m}^{-2}$, consistent with the prediction of Ref. [20] as required for the confinement of a particle beam to an optical-scale accelerator, is employed in order to confine the particles in the $y$ dimension, as shown in Fig. 5(c). The left plots in Fig. 5 correspond to a nontapered structure and produce only an energy modulation of the particles, whereas the right case corresponds to a resonant acceleration with a reduced bucket size shown by the red separatrix in Fig. 5(a). The Hamiltonian for a resonantly tapered accelerator can be written as $H(\phi, \delta)=\left(\beta_{r}^{2} / 2 \gamma_{r}\right) \delta^{2}+\alpha_{0}\left[\cos \phi+\phi \sin \phi_{r}+1\right]$, where $\delta=\left(\gamma \beta-\gamma_{r} \beta_{r}\right) / \gamma_{r} \beta_{r}$ is the fractional momentum deviation. In Fig. 5(a), the Hamiltonian phase space level sets are superimposed on the plot for comparison, showing that for the nontapered structure (left column) the "accelerating bucket" inside the Hamiltonian separatrix shown in red extends over all phases, whereas in the plots for the tapered structure (right column), the separatrix corresponds to a limited region of injection phases that produce net acceleration.

Referring to the right column in Fig. 5(b), it is clearly seen that particles with the initial phase in the segment within the bucket region marked by the vertical dashed lines gain substantial energy $(T>79 \mathrm{KeV})$. This segment corresponds to the phase acceptance range of the structure. As seen in Fig. 5(a), the maximal fractional energy gain is attained when the synchronicity condition is met, meaning that the final captured phase $\phi=\phi_{r}=3 \pi / 4$. In Fig. 5(b), which is plotted as a function of initial injection phase $\phi_{0}$, we see that this maximum energy $(\sim 100 \mathrm{keV})$ corresponds to particles that were initially on the left side of the bucket region (near $\phi_{0}=1$ ). It should be emphasized that the maximal energy loss $(\sim 7 \mathrm{keV})$ for particles outside the phase acceptance region is substantially smaller than the maximal energy gain $(\sim 20 \mathrm{keV})$. This effect is due to the tapering of the structure and means that a particle beam uniformly distributed in phase will exhibit net acceleration. The tapering maintains synchronicity only for particles in the vicinity of the resonance and renders the deceleration process less efficient than the acceleration process.

For the constant phase velocity case $\left(\beta_{r}=0.5\right)$ shown on the left, the particles experience merely an energy modulation. This scenario corresponds with most experiments conducted to date using laser-driven microstructures of this sort, in which the width of the resultant energy modulation is then used to extrapolate the accelerating gradient $G_{0}$. By contrast, for the tapered phase velocity case in the right column in Fig. 5, there is a distinct population of accelerated electrons within the Hamiltonian separatrix (in red) representing the captured population.

We can use this simplified resonant particle model to predict capture efficiency by defining a "trapping fraction" as the fraction of particles within $10 \%$ of the final resonant kinetic energy $T_{f}=m c\left(\gamma_{f}-1\right)$. By integrating the equations of motion for a particle distribution covering uniformly all possible injection phases $\phi_{0}=\phi(z=0)$ from 0 to $2 \pi$ and resonant phases $\phi_{r}$ from 0 to $\pi$, we obtain the trapping fraction and kinetic energy distributions depicted in Fig. 6. To obtain these results, we assume a device length of $L=17 \lambda_{p}$, the driving laser wavelength is $\lambda=2 \mu \mathrm{m}$, and the effective accelerating field is $G_{0}=1.4 \mathrm{GV} / \mathrm{m}$. 
(a)



(b)



FIG. 6. Predicted trapping fraction (a) and total energy gain (b) as functions of resonant phase $\phi_{r}$ in a tapered MLA structure.

The initial particle relative velocity is $\beta_{0}=0.5$ (corresponds to an initial kinetic energy of $T_{0}=79 \mathrm{KeV}$ ). As can be seen in Fig. 6(a), the capture efficiency is minimal at $\phi_{r}=\pi / 2$, corresponding to the minimum bucket width. Operating at other resonant phases away from $\pi / 2$ can increase the size of the accelerating bucket, thereby improving the capture efficiency at the expense of the accelerating gradient. Hence, when designing the laser accelerator, there is a fundamental tradeoff between attaining the maximal energy gain versus increasing the capture efficiency.

We have considered the two resonant phase examples in Fig. 5 for purely illustrative purposes. The MLA structure in Sec. II is optimized for a resonant phase of $\phi_{r}=\pi / 2$. As seen in Fig. 6, this choice corresponds to the maximum gradient and thus maximum energy gain, which may aid anticipated future experimental demonstrations by providing a better energy contrast between accelerated and nonaccelerated particles. In Secs. IV-VI, we study this example with particle tracking studies and compare to the resonant particle model of Eqs. (7) and (8).

\section{LONGITUDINAL DYNAMICS FOR A LONG BUNCH WITH NO SPACE CHARGE}

Simulations of the MLA structure using a 3D FDTD code were carried out to obtain a complete mapping of the electromagnetic field in the structure. This field distribution allows for simulating the trajectories of particles propagating through the structure. We use the particle tracking code General Particle Tracer (GPT) to simulate the propagation of a beam of electrons through the enclosed (dual-sided) MLA configuration discussed in Sec. II.

The simulated beam contains $5 \times 10^{5}$ particles with an initial energy of $79 \mathrm{keV}$, corresponding to an initial velocity of $\beta_{0}=0.5$, with a Gaussian distribution over the aperture of the structure with root-mean-square (rms) widths in $x$ and $y$ of $32 \mathrm{~nm}$ and a normalized transverse emittance of $0.01 \mathrm{~nm}$. The temporal rms duration of the beam is chosen to be $50 \mathrm{fs}$, corresponding to a spatial length of $7.5 \mu \mathrm{m}$. The beam parameters were chosen to mimic a stream of particles from, e.g., an electron microscope test beam, such as those employed in Refs. [6,7]. As the resulting density of particles here greatly exceeds that of a realistic

electron microscope beam by orders of magnitude, spacecharge effects between particles are neglected in the simulation (see Sec. VI for the impact of space-charge effects). We note that simulations of a longer temporal duration, low-density, beam such as would be used in a demonstration experiment would greatly increase the required simulation time without adding substantially different physics.

Figure 7 depicts the calculated final phase space distribution of the beam after exiting the MLA structure described in Sec. II, with the GPT simulations on the left, and the resonant particle model, integrating Eqs. (7) and (8), on the right. Figure 7(a) indicates that the longitudinal phase space distribution of the beam has been severely distorted into a nearly periodic arrangement spanning from low to high energy. Bunches of particles at the top of the plot correspond to the captured populations in successive accelerating "buckets" or phase space periods of the accelerating wave, giving rise to a sharp spike at the final accelerated energy $T=103 \mathrm{keV}$ in the energy spectrum plot in Fig. 7(c). To facilitate comparison with the solutions of Eqs. (7), we focus in Fig. 7(b) on the final captured particle population at the back end of the bunch just as it is exiting from the accelerating field region. This range extends from $z=L$ to $z=L+\lambda_{p}$. The captured population is plotted against the corresponding final phase (a)

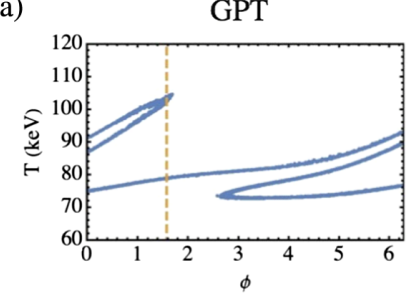

(b)

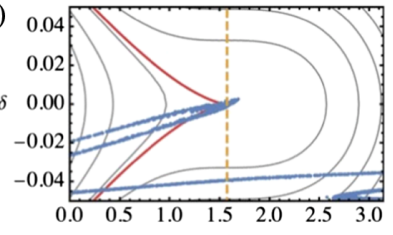

(c)

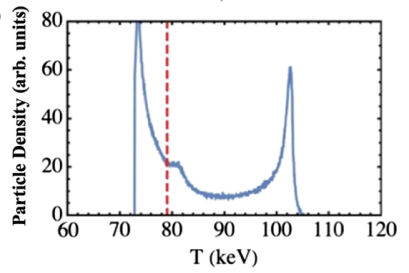


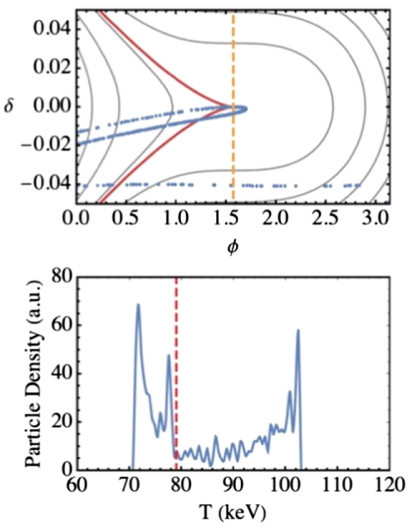

FIG. 7. Final phase space distribution over one structure period of a GPT simulated beam immediately after exiting the tapered MLA including (a) the final beam energy vs phase distribution, (b) the phase space plot superimposed with Hamiltonian level sets and sepatratrix (in red), and (c) the corresponding energy spectrum of the full beam with the initial energy marked in red. Left column, GPT particle tracking results; right column, resonant particle model from Sec. III. 
$\phi=k_{p} z$, where $\lambda_{p}$ and $k_{p}$ are evaluated at $z=L$. The resulting phase space plot and energy spectrum in Fig. 7(c) on the left agree remarkably well with the predictions of the analytic (continuous) model shown on the right. We note that in Fig. 7 (left) we have included a transformation on the final phase values of the form $\phi \rightarrow \phi-k_{p} \eta \delta$, where $\eta=$ $L / 2 \gamma_{r}^{2}$ and $\delta=\left(\gamma-\gamma_{r}\right) / \gamma_{r}$ to account in an approximate way for the average longitudinal velocity bunching of the particles. This produces a slight clockwise shearing of the distribution, which aids comparison with the results of the analytical calculation.

\section{MICROBUNCHING}

Velocity bunching produced by the energy difference between the accelerated and decelerated particles results in a lateral tilting of the longitudinal $(T-z$ or $\delta-\phi)$ phase space distributions in successive buckets which increases from the tail to the head of the bunch. In addition, particles positioned at larger values of $z$ have also propagated for a longer time, thus extending the spatial separation between the more energetic and the less energetic population. As mentioned above, this effect was included in the results in Fig. 7 (left) by an approximate phase space transformation on the phase variable $\phi$. Consequently, there is an intermediate value of $z$ at which the population of the corresponding phase space bucket is vertical and both the low- and high-energy particles overlap spatially in $z$, thus producing a strong longitudinal density modulation or microbunching of the beam. This highlights that even an initially nonbunched beam will be microbunched at the structure periodicity. A prebuncher to inject a beam already microbunched in this way could be used as a way to improve the capture efficiency in the accelerator.

A common method of quantifying the microbunching effect is to calculate the Fourier coefficient of the beam distribution that corresponds to the fundamental period $\left(\lambda_{p}\right)$. Defining the complex Fourier coefficients $c_{n}(z)=\frac{1}{2 \pi} \int_{0}^{2 \pi} \rho(\phi, z) e^{-i n \phi} d \phi$, the fundamental component corresponds to $n=1$. Here $\rho(\phi, z)$ is the computed longitudinal density of the bunch at phase $\phi$ after propagating a distance $z$. Figure 8(a) depicts the longitudinal phase space distribution of the particles at an intermediate position inside the MLA such that the particle distribution spans the length of the accelerator from $z=0$ to $z=L \approx 18 \mu \mathrm{m}$. The magnitude of the normalized bunching coefficient $\left|c_{1} / c_{0}\right|$ obtained from the solutions of Eq. (7) is shown in blue in Fig. 8(b) as a function of the longitudinal coordinate $z$. Note that, based on the resonant particle model, it is expected that the microbunching effect would be maximal around $z=8 \mu \mathrm{m}$. The longitudinal number density $d N / d z$ of the beam (normalized to the total number of particles in the simulation $N_{b}$ ) obtained from the distribution in Fig. 8(a) is superimposed in red in Fig. 8(b). The prediction of the optimal bunching point is confirmed (a)

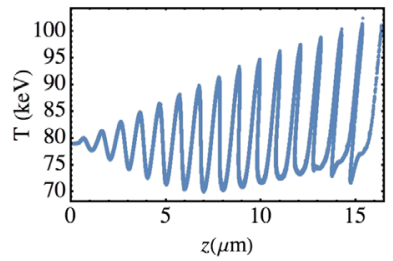

(b)

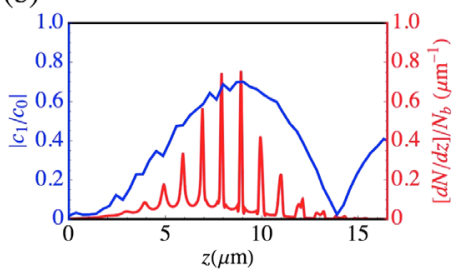

FIG. 8. Plots of the (a) longitudinal phase space distribution from GPT tracking of a long (50 fs) uniformly distributed bunch; (b) bunching factor (blue) predicted from the resonant particle model and the corresponding density profile (red) of the distribution from (a) as a function of propagation distance $z$. The bounds of the plots in $z$ correspond to the entrance and exit of the tapered MLA.

by the GPT simulations, which show the strongest and narrowest density peaks near the peak value of the bunching coefficient, with a minimum full width at half maximum (FWHM) bunch duration of approximately 500 as. A comparison with Fig. 8(a) indicates that the optimal bunching point corresponds to an overlap of the low- and high-energy parts of the distribution within a single period. Note that the appearance of a secondary bunching peak in Fig. 8(b) between $z=12$ and $z=15 \mu \mathrm{m}$ is caused by overlapping of low-energy particles from a preceding bucket with high-energy particles belonging to the following one. These secondary peaks are also responsible for the spurious increase in the bunching factor (blue) in Fig. 8(b) for $z>14 \mu \mathrm{m}$. The formation of this effect can be seen in the rightmost bunches in Fig. 8(a). As the bunches continue to propagate, the lower-energy (uncaptured) particles flow into the phase space regions of adjacent buckets as was also seen in Fig. 7(a), and the modulation of the beam density is eventually washed out. This suggests the possibility of using a shorter section of MLA structure, which terminates at the point of optimal bunching, as a prebuncher to improve the initial capture efficiency in a subsequent longer MLA device.

\section{BEAM LOADING EFFICIENCY}

The characteristic impedance of the structure can be defined as $Z_{C}=G_{0}^{2} \lambda^{2} / P$, where $P=E_{0}^{2} \lambda D / 2 Z_{0}$ is the peak laser power over a single optical period, assuming a Gaussian laser beam profile with free space impedance $Z_{0}=377 \Omega$. Relative to the coordinates in Fig. $1, D$ is the illumination size in $x$, which is transverse to the electron axis $z$. The resulting coupling efficiency will thus depend upon the laser focal spot size at the structure. Combining these expressions gives

$$
Z_{C}=2 Z_{0} \kappa^{2} \frac{\lambda}{D}
$$

where $\kappa=G_{0} / E_{0}$ is the so-called "structure factor." To allow the full illumination of a single transverse period, we 
take $D=\lambda$ as a reasonable value. The optimal bunch charge and efficiency of the accelerator may then be written [21] as $q_{\mathrm{opt}}=G_{0} /\left(2 c Z_{H} / \lambda^{2}\right)$ and $\eta_{\mathrm{opt}}=L Z_{C} /\left(4 c \tau Z_{H}\right)$, respectively, where $Z_{H}$ is the Cherenkov wake impedance, which is a function of the structure's material properties and geometry, and $\tau$ is the laser pulse duration. A conservative estimate of $Z_{H}$ can be obtained from Ref. [22] for a flat (2D planar) geometry: $Z_{H}=\pi Z_{0} \lambda^{2} /\left(16 a^{2}\right)$. Here $a$ is the half width of the channel. From the form of Eq. (10), we see that, for a given structure length and pulse duration, the efficiency of the accelerator scales as $\kappa^{2}$. For the MLA structure design considered here, $\kappa=1.34$. By comparison, $\kappa$ is lower than unity for other laser-driven structure topologies that have been considered $[1,7]$. The plasmonic field enhancement of the MLA thus potentially allows for increased efficiency compared with some other approaches. For a wavelength of $\lambda=2 \mu \mathrm{m}, 100$ fs FWHM laser pulse duration (corresponding to $\tau=75 \mathrm{fs}$ ), and a channel width $a=100 \mathrm{~nm}$, we obtain a characteristic impedance $Z_{C}=1313 \Omega$. For the gradient assumed in our calculations above, $G_{0}=1.4 \mathrm{GeV} / \mathrm{m}$, the corresponding optimal charge and efficiency are $q_{\mathrm{opt}}=0.3 \mathrm{fC}$ (1970 electrons) and $\eta_{\mathrm{opt}}=1.6 \%$, respectively, for single-bunch operation. For multibunch operation, the efficiency can be increased to several tens of percent [21]. In the following section, we calculate the transport and external focusing requirements for confinement to the channel of half width $a=100 \mathrm{~nm}$ for a bunched electron beam of this charge.

\section{BUNCHED BEAM WITH SPACE CHARGE}

We consider now the case where a prebunched beam is injected into the MLA. We assume a single bunch with a rms bunch duration of 100 as, which corresponds to $5.4^{\circ}$ of laser phase and is similar in order of magnitude to achieved optical scale bunching techniques [23]. A round beam is assumed that is Gaussian in the transverse and longitudinal profile with a rms size of $\sigma_{x, y}=32 \mathrm{~nm}$ and initial normalized transverse emittances $\epsilon_{n x, y}=0.1 \mathrm{~nm}$, consistent with achievable emittances of compatible tip-based field emission sources [24]. In accordance with the beam loading efficiency argument in Sec. VI, we assume an optimal bunch charge of 2000 electrons. As compared with the long-bunch case considered in Sec. IV, such a microbunched beam experiences significant internal spacecharge forces which lead to substantial emittance growth and must be mitigated by some form of external focusing. In the GPT tracking simulation, we assume an external solenoid field with a peak normalized field strength given by $K^{2}=e B /(2 m c \beta \gamma)$, where $B$ is the peak magnetic field on axis.

As seen in Fig. 9, the emittance is nearly compensated for by a focusing strength of $K=2 \times 10^{-6} \mathrm{~m}^{-1}$, at which point the number of transmitted particles reaches $100 \%$. We note that the emittance values on the far left inf Fig. 9(a)
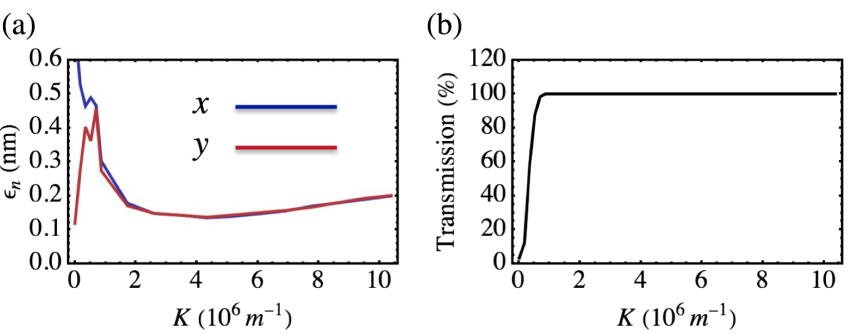

FIG. 9. Plots of the (a) normalized emittance and (b) transmitted fraction of particles from GPT tracking of a short (100 as) Gaussian bunch with 2000 electrons, as a function of focusing strength parameter $K$ corresponding to a solenoidal field centered on the tapered MLA.

display sharp peaks due to the fact that very few electrons are transmitted when the focusing strength is near zero, making the emittance calculation statistically noisy. The focusing required for emittance preservation is a factor of 4 higher than the value of $K^{2}=1 \mu \mathrm{m}^{-2}$ estimated using the simple analytical model for the long-bunch space-chargefree case in Sec. III. We note that this focusing corresponds to a peak axial magnetic field value of $4 \mathrm{kT}$, which is well beyond the capability of conventional solenoid magnets. In practice, focusing of this magnitude in the MLA or another optical-scale accelerator requires the implementation of focusing techniques where the laser field itself provides a compensating transverse electromagnetic force. Schemes for accomplishing this using harmonic focusing and alternating phase focusing have been proposed in Refs. [25,26] and are currently being implemented in various laser-driven accelerator designs.

\section{CONCLUSIONS}

We have proposed a design for a laser-driven accelerator with a tapered phase velocity for matching to a subrelativistic electron beam. The proposed structure utilizes a pair of parallel plasmonic metasurfaces for focusing the driving laser field and generating a series of accelerating "hot spots" inside of a narrow vacuum gap between the plates. We have presented an electromagnetic design for a 17-cell structure for $79 \mathrm{keV}$ injected electrons which supports a gradient of $1.4 \mathrm{GeV} / \mathrm{m}$ under dual illumination by lasers with a $0.5 \mathrm{GV} / \mathrm{m}$ incident field, consistent with laser preliminary damage measurements conducted on prototype devices fabricated with gold layers onto fused silica. We have conducted particle tracking studies through a simulated 3D field map of the tapered device. We find that, for a long-bunch scenario with no space charge, the phase space dynamics are well explained by a standard resonant particle model of the beam dynamics using linearized fields in the accelerating channel and provide a prediction of the external focusing required for transverse confinement. We also observe microbunching of the initially nonbunched beam in the longitudinal phase space that is consistent with 
predictions based on the bunching factor obtained from the resonant particle model. However, for a prebunched beam that occupies only a few degrees of optical phase and contains 2000 electrons (corresponding to the estimated optimal beam-loaded charge), the space-charge forces within the beam are found to be substantial and require approximately 4 times larger focusing strength than for the nonbunched case. We note, however, that, although the optimal beam-loaded charge is assumed for evaluation of the space-charge effect, wakes in the structure are not explicitly modeled in the tracking code. In reality, for bunched operation, the beam-loaded gradient would need to be built into the tapered structure design and wakefields appropriately included in the tracking code. A detailed evaluation of wake effects in laser-driven microstructure accelerators is beyond the scope of the present paper but remains an important subject for future studies.

Compared to equivalent dielectric laser-driven accelerators, the proposed MLA structure exhibits two important advantages: (i) the plasmonic metasurfaces are thin $(\sim 30-40 \mathrm{~nm})$ and can be relatively easily patterned with the subwavelength features needed for operating at the fundamental spatial harmonic of the driving field; (ii) the metasurface's ability to create a plasmonic field enhancement increases the efficiency of the acceleration process and renders the design simpler. Although the maximal acceleration gradient that can be obtained by the MLA is not necessarily greater that those of DLA structures (this is determined primarily by the material damage threshold), the MLA is more efficient and exhibits a larger normalized acceleration gradient, or structure factor. Combined with the relatively simple fabrication requirements, these properties render the proposed MLA structure highly attractive for a compact and efficient accelerator system, especially for subrelativistic particles.

\section{ACKNOWLEDGMENTS}

This research was supported by the Binational Science Foundation Grant No. 2014725, U.S. National Science Foundation Grant No. 1535711, by the U.S. Department of Energy under Grant No. DE-AC02-76SF00515, and by the Gordon and Betty Moore Foundation (GBMF4744).

\section{APPENDIX A: MLA UNIT-CELLS DESIGN AND PARAMETERS}

Table 1 lists the unit-cell and the slit nanoantenna dimensions of the MLA structure design, based on Eq. (2). The table also lists the corresponding values of the resonant particle relativistic velocity and kinetic energy according to Eq. (1). This structure was used for the GPT simulations described in Secs. IV-VI.
TABLE I. Optimized dimensions for a 17-cell MLA.

\begin{tabular}{lccccc}
\hline \hline Cell & $\lambda_{p}[\mu \mathrm{m}]$ & $S_{H}[\mathrm{~nm}]$ & $S_{W}[\mathrm{~nm}]$ & $\beta$ & $\mathrm{KE}[\mathrm{keV}]$ \\
\hline 1 & 1.00 & 200 & 600 & 0.501 & 79.0 \\
2 & 1.01 & 200 & 600 & 0.506 & 80.9 \\
3 & 1.02 & 200 & 600 & 0.510 & 82.8 \\
4 & 1.03 & 200 & 600 & 0.515 & 84.7 \\
5 & 1.04 & 200 & 600 & 0.520 & 86.7 \\
6 & 1.05 & 200 & 600 & 0.524 & 88.7 \\
7 & 1.06 & 200 & 600 & 0.529 & 90.7 \\
8 & 1.07 & 200 & 600 & 0.534 & 92.8 \\
9 & 1.08 & 200 & 600 & 0.538 & 94.9 \\
10 & 1.09 & 200 & 600 & 0.543 & 97.0 \\
11 & 1.09 & 200 & 600 & 0.547 & 99.2 \\
12 & 1.10 & 200 & 600 & 0.552 & 101.4 \\
13 & 1.11 & 200 & 600 & 0.557 & 103.6 \\
14 & 1.12 & 200 & 600 & 0.561 & 105.9 \\
15 & 1.13 & 200 & 600 & 0.566 & 108.2 \\
16 & 1.14 & 200 & 600 & 0.570 & 110.6 \\
17 & 1.15 & 200 & 600 & 0.575 & 112.9 \\
\hline \hline
\end{tabular}

\section{APPENDIX B: FILED PROFILE IN A PERIODIC MLA STRUCTURE}

We consider a planar symmetric geometry in a fixed Cartesian reference frame that is invariant in the $x$ coordinate, periodic in $z$ with periodicity $\mathbf{u}=\lambda_{p} \hat{\mathbf{z}}$, and with varying dielectric constant $\epsilon(y, z)$. Under illumination by a plane wave of amplitude $E_{0}$ propagating with wave number $\mathrm{k}_{i}$ in the $y-z$ plane, a set of longitudinal Floquet modes are permitted with wave numbers $k_{n}=k_{0}+n k_{p}$, where $k_{0}=$ $\left|\mathbf{k}_{i} \cdot \mathbf{u}\right|=(\omega / c) \sqrt{\epsilon_{i}} \cos \theta$ is the projection of the incident plane wave onto the fundamental periodicity, $k_{p}=2 \pi / \lambda_{p}$, $\epsilon_{i}$ is the dielectric constant in the region of the incident field, and $\theta$ is the incidence angle of the laser.

For a $P$-polarized field (i.e., $E_{0}$ lies in the $y$ - $z$ plane) incident from the region $y<0$ and propagating in the positive $y$ direction onto such a periodic structure occupying the upper half-space $(y>0)$, the nonvanishing electric field components in the vacuum region $y<0$ are given by [27]

$$
\begin{gathered}
E_{y}=-E_{0} \sum_{n} \frac{k_{n}}{\Lambda_{n}}\left[\delta_{n, 0} e^{i \Lambda_{n} y}-r_{n} e^{-i \Lambda_{n} y}\right] e^{i k_{n} z}, \\
E_{z}=E_{0} \sum_{n}\left[\delta_{n, 0} e^{i \Lambda_{n} y}+r_{n} e^{-i \Lambda_{n} y}\right] e^{i k_{n} z},
\end{gathered}
$$

where $\delta$ is the Kronecker delta function, $r_{n}$ and $\Lambda_{n}$ are the reflection coefficient and transverse wave number, respectively, for Floquet mode $n$, and we suppress the explicit harmonic time dependence for convenience. We note that the delta function term corresponds to the incident plane wave $E_{0} e^{i \mathbf{k}_{i} \cdot \mathbf{r}}$ with the association $\mathrm{k}_{i}=\Lambda_{0} \hat{\mathbf{y}}+k_{0} \hat{\mathbf{z}}$. With the addition of a second identical periodic structure in the lower $(y<0)$ half-space but mirror reversed about $y=0$ and the introduction of a symmetric vacuum gap region between 
the two periodic structures, the transverse propagating waves in (B1) and (B2) will experience infinitely many internal reflections within the vacuum region, giving rise to general coefficients $C_{n}$ and $D_{n}$ for the forward and reverse components:

$$
\begin{gathered}
E_{y}=-E_{0} \sum_{n} \frac{k_{n}}{\Lambda_{n}}\left[C_{n} e^{i \Lambda_{n} \mathrm{y}}-D_{n} e^{-i \Lambda_{n} \mathrm{y}}\right] e^{i k_{n} z}, \\
E_{z}=E_{0} \sum_{n}\left[C_{n} e^{i \Lambda_{n} \mathrm{y}}+D_{n} e^{-i \Lambda_{n} \mathrm{y}}\right] e^{i k_{n} z} .
\end{gathered}
$$

Because of the symmetry of the geometry about the $x-z$ plane, an identical laser incident from above (same incidence angle and amplitude) and propagating toward the negative $y$ direction gives rise to an identical mode but with the reflection coefficients interchanged $\left(C_{n} \leftrightarrow D_{n}\right)$. Adding this solution to that above and noting that for longitudinal phase velocities lower than the speed of light the transverse wave number $\Lambda_{n}$ is imaginary, we make the substitution $\Gamma_{n} \equiv i \Lambda_{n}$ and define $a_{n} \equiv 2\left(C_{n}+D_{n}\right)$. The transverse $(y)$ exponential terms then add to form hyperbolic functions, and the form of Eqs. (3)-(5) is immediately obtained, with the inclusion of an arbitrary phase factor of $-i$. The magnetic field component [Eq. (5)] can be directly derived from the electric field using Faraday's law in a source-free region with harmonic time dependence: $\nabla \times \mathbf{E}=i(\omega / c) \mathbf{B}$.

\section{APPENDIX C: LASER INDUCED DAMAGE TESTS IN MLA STRUCTURES}

In order to gauge a reasonable incident field level $E_{0}$ for the simulations of Secs. IV-VI, laser-induced damage was measured on a series of single-sided MLA arrays. The samples were fabricated by depositing a 40-nm-thick gold layer onto a fused silica substrate and then etching the gold using a focused ion beam (FIB) in a sequence of patterned arrays based upon the geometrical design outlined in Sec. II. Damage was induced using a pulsed laser with a wavelength of $2 \mu \mathrm{m}$, a $35 \mu \mathrm{m}$ spot $\left(1 / e^{2}\right.$ diameter), a pulse duration of $250 \mathrm{fs}$, and a repetition rate of $100 \mathrm{kHz}$. Successive MLA arrays were exposed at increasing pulse energies for $100 \mathrm{~s}$ (corresponding to 10 million shots) per damage site.

The samples were analyzed by inspection with an optical microscope. Several examples are shown in Fig. 10, with increasing pulse energy from left to right and corresponding incident field intensity detailed in the caption. Because of the operational range of the laser power meter used, the lowest reliably measured pulse energy was that in Fig. 10(b), which corresponds to an incident field $E_{0}=0.54 \mathrm{GV} / \mathrm{m}$, corresponding to a fluence of $10 \mathrm{~mJ} / \mathrm{cm}^{2}$. Since some scattered damage was still observed at this intensity, it provides only an upper limit on the observed damage threshold. However, we note that, since the damage fluence is relatively insensitive to pulse duration $\tau$ in this pulse length regime, in principle, a shorter (a)

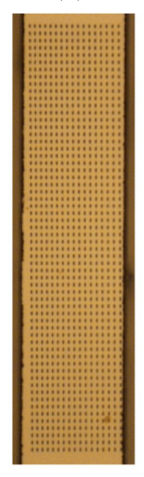

(b)

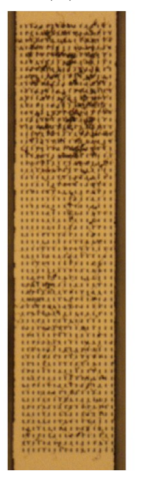

(c)



(d)

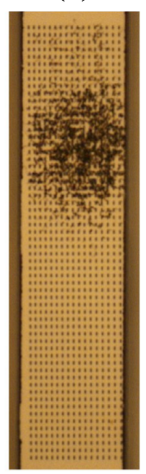

FIG. 10. Example optical microscope images of single-sided MLA arrays after timed exposures of $10^{7}$ laser shots with incident field strengths corresponding to (a) 0 (laser off), (b) 0.54 , (c) 0.77 , and (d) $0.91 \mathrm{GV} / \mathrm{m}$, illustrating the onset and progression of damage.

laser pulse would increase the corresponding sustainable incident field as $\tau^{-1 / 2}$. Consequently, using a $50 \mathrm{fs}$ pulse instead could increase the sustainable $E_{0}$ by a factor of 2.23. It has recently been shown that, even with such short pulses, acceleration in an optical structure over hundreds of periods can be achieved by tilting the laser pulse [28].

[1] T. Plettner, P. P. Lu, and R. L. Byer, Proposed few-optical cycle laser-driven particle accelerator structure, Phys. Rev. ST Accel. Beams 9, 111301 (2006).

[2] K. P. Wootton, Z. Wu, B. M. Cowan, A. Hanuka, I. V. Makasyuk, E. A. Peralta, K. Soong, R. L. Byer, and R. Joel England, Demonstration of acceleration of electrons at a dielectric microstructure using femtosecond pulses, Opt. Lett. 41, 2696 (2016).

[3] E. A. Peralta, K. Soong, R. J. England, E. R. Colby, Z. Wu, B. Montazeri, C. McGuinness, J. McNeur, K. J. Leedle, D. Walz, E. B. Sozer, B. Cowan, B. Schwartz, G. Travish, and R. L. Byer, Demonstration of electron acceleration in a laser-driven dielectric microstructure, Nature (London) 503, 91 (2013).

[4] R. J. England, Review of laser-driven photonic structurebased particle acceleration, IEEE J. Sel. Top. Quantum Electron. 22, 4401007 (2016).

[5] T. Plettner, R. L. Byer, and B. Montazeri, Electromagnetic forces in the vacuum region of laser-driven layered grating structures, J. Mod. Opt. 58, 1518 (2011).

[6] J. Breuer and P. Hommelhoff, Laser-Based Acceleration of Nonrelativistic Electrons at a Dielectric Structure, Phys. Rev. Lett. 111, 134803 (2013).

[7] K. J. Leedle, R. F. Pease, R. L. Byer, and J. S. Harris, Laser acceleration and deflection of $96.3 \mathrm{keV}$ electrons with a silicon dielectric structure, Optica 2, 158 (2015).

[8] K. J. Leedle, A. Ceballos, H. Deng, O. Solgaard, R. F. Pease, R. L. Byer, and J. S. Harris, Dielectric laser acceleration of sub-100 keV electrons with silicon dual-pillar grating structures, Opt. Lett. 40, 4344 (2015). 
[9] C.-M. Chang and O. Solgaard, Silicon buried gratings for dielectric laser electron accelerators, Appl. Phys. Lett. 104, 184102 (2014).

[10] D. Bar-Lev and J. Scheuer, Plasmonic metasurface for efficient ultrashort pulse laser-driven particle acceleration, Phys. Rev. ST Accel. Beams 17, 121302 (2014).

[11] C. Joshi, Laser accelerators, IEEE Trans. Nucl. Sci. 32, 1576 (1985).

[12] J. Rosenzweig, A. Murokh, and C. Pellegrini, A Proposed Dielectric-Loaded Resonant Laser Accelerator, Phys. Rev. Lett. 74, 2467 (1995).

[13] L. W. Alvarez, H. Bradner, J. V. Franck, H. Gordon, J. Donald Gow, L. C. Marshall, F. Oppenheimer, W. K. H. Panofsky, C. Richman, and J.R. Woodyard, Berkeley proton linear accelerator, Rev. Sci. Instrum. 26, 111 (1955).

[14] I. M. Kapchinskiy and V. A. Teplyakov, Prib. i Techn. Exper. 2, 19 (1970).

[15] U. Niedermayer, O. Boine-Frankenheim, and T. Egenolf, Designing a dielectric laser accelerator on a chip, J. Phys. Conf. Ser. 874, 012041 (2017).

[16] T. W. Ebbesen, H. J. Lezec, H. F. Ghaemi, T. Thio, and P. A. Wolff, Extraordinary optical transmission through sub-wavelength hole arrays, Nature (London) 391, 667 (1998).

[17] J. McNeur, M. Kozak, D. Ehberger, N. Schonenberger, A. Tafel, A. Li, and P. Hommelhoff, A miniaturized electron source based on dielectric laser accelerator operation at higher spatial harmonics and a nanotip photoemitter, J. Phys. B 49, 034006 (2016).

[18] L. Smith, Linear accelerators, in Encyclopedia of Physics (Springer, Berlin, 1959), Vol. 8, pp. 341-389.
[19] Linear Accelerators, edited by P. M. Lapostolle and A. L. Septier (North-Holland, Amsterdam, 1969), Chap. 2, pp. 19-27.

[20] R. J. England et al., Dielectric laser accelerators, Rev. Mod. Phys. 86, 1337 (2014).

[21] Y.C. Neil Na, R. H. Siemann, and R. L. Byer, Energy efficiency of an intracavity coupled laser-driven linear accelerator pumped by an external laser, Phys. Rev. ST Accel. Beams 8, 031301 (2005).

[22] K. Bane and G. Stupakov, Using surface impedance for calculating wakefields in flat geometry, Phys. Rev. ST Accel. Beams 18, 034401 (2015).

[23] C. M. S. Sears et al., Production and characterization of attosecond electron bunch trains, Phys. Rev. ST Accel. Beams 11, 061301 (2008).

[24] K. L. Jensen, P. G. O'Shea, D. W. Feldman, and J. L. Shaw, Emittance of a field emission electron source, J. Appl. Phys. 107, 014903 (2010).

[25] U. Niedermayer, T. Egenolf, O. Boine-Frankenheim, and P. Hommelhoff, Alternating phase focusing for dielectric laser acceleration, arXiv:1806.07287.

[26] B. Naranjo, A. Valloni, S. Putterman, and J. B. Rosenzweig, Stable Charged-Particle Acceleration and Focusing in a Laser Accelerator Using Spatial Harmonics, Phys. Rev. Lett. 109, 164803 (2012).

[27] L. Pilozzi, A. D’Andrea, and R. Del Sole, Electromagnetic properties of a dielectric grating. I. Propagating, evanescent, and guided waves, Phys. Rev. B 54, 10751 (1996).

[28] D. Cesar, J. Maxson, P. Musumeci, X. Shen, R. J. England, K. P. Wootton, and S. Tan, Enhanced energy gain in a dielectric laser accelerator using a tilted pulse front laser, Opt. Express 26, 29216 (2018). 